\title{
Identities involving skew Lie product and a pair of generalized derivations in prime rings with involution
}

\author{
B. Bhushan, G. S. Sandhu and D. Kumar
}

\begin{abstract}
In this paper, we consider skew Lie product on an involutive ring and study several algebraic identities for it, which include generalized derivations of the ring. The results give information about the commutativity of the ring and a description of the generalized derivations.
\end{abstract}

Key Words: Generalized derivations, involution, prime ring Mathematics Subject Classification 2010: 16W10, 16N60, 16W25

\section{Introduction}

In this article, $R$ denotes an associative prime ring with center $Z(R)$, unless otherwise mentioned. For any $x, y \in R$, the symbol $[x, y]$ (resp., $x \circ y$ ) denotes the commutator (resp., anti-commutator) $x y-y x$ (resp., $x y+y x)$. If for any $a, b \in R, a R b=(0)$ implies either $a=0$ or $b=0$, then $R$ is said to be a prime ring. For any $n \in \mathbb{Z}^{+}, R$ is called n-torsion free if for any $x \in R$, $n x=0$ implies $x=0$.

Let us recall that an involutive ring $R$ is a ring with an additional unary operation $*$, called involution, such that $(a+b)^{*}=a^{*}+b^{*},(a b)^{*}=b^{*} a^{*}$, and $\left(a^{*}\right)^{*}=a$ for all $a, b \in R$. An element $x \in R$ is called symmetric element if $x^{*}=x$ and it is called skew symmetric if $x^{*}=-x$. The sets of symmetric and skew-symmetric elements in $R$ are denoted by $H(R)$ and $S(R)$, respectively. The involution ' $*$ ' is called involution of the first kind if $Z(R) \subseteq H(R)$, otherwise it is of the second kind. Moreover, $S(R) \cap Z(R) \neq(0)$, in case the involution is of the second kind. Further, if $R$ is 2-torsion free, then for each $x \in R$, we have a unique representation $2 x=h+k$, where $h \in H(R)$ and $k \in S(R)$. In such case, $x$ is normal, i.e., $x x^{*}=x^{*} x$ if and only if $h k=k h$. A ring in which all elements are normal is called normal ring, e.g., the ring of quaternions. 
An additive mapping $d: R \rightarrow R$ is called a derivation if $d(x y)=d(x) y+$ $x d(y)$ for all $x, y \in R$. An example of derivation is the so called inner derivation, e.g., the mapping $x \mapsto[a, x]$ where $a \in R$ is a fixed element. By generalized inner derivation we mean an additive mapping $F: R \rightarrow R$ such that for fixed elements $a, b \in R, F(x)=a x+x b$ for all $x \in R$. More generally, an additive mapping $F: R \rightarrow R$ which is uniquely determined by a derivation $d: R \rightarrow R$ such that $F(x y)=F(x) y+x d(y)$ for all $x, y \in R$, is called generalized derivation (see [8]). In particular, if $d=0$, then $F$ is called a left centralizer.

In [13], Herstein proved that if $R$ is a 2-torsion free prime ring and $d$ is a non-zero derivation of $R$ such that $[d(x), d(y)]=0$ for all $x, y \in R$, then $R$ is commutative. Later, Daif [10] extended this result for two sided ideals of a semiprime ring. In [7], Bell and Rehman generalized this classical theorem to the class of generalized derivations of prime rings. Recently, Dar and Ali [11] examined this situation on a 2-torsion free prime ring with involution of the second kind. Precisely, they proved the following: Let $R$ be a 2-torsion free prime ring with involution of the second kind and $d$ be a non-zero derivation of $R$ such that $\left[d(x), d\left(x^{*}\right)\right]=0$ for all $x \in R$. Then $R$ is commutative. Motivated by these results, Ali et al. [5] proved the following: Let $R$ be a non-commutative 2-torsion free prime ring with involution of the second kind. If $R$ admits a non-zero generalized derivation $F: R \rightarrow R$ such that $\left[F(x), F\left(x^{*}\right)\right]=0$ for all $x \in R$, then $R$ is an order in a central simple algebra of dimension at most 4 over its center and $F(x)=a x+x b$ for all $x \in R$ for fixed $a, b \in U$ such that $a-b \in C$.

A mapping $f: R \rightarrow R$ is called commutativity preserving on $R$ if $[x, y]=0$ implies $[f(x), f(y)]=0$ for all $x, y \in R$. More generally, $f$ is called strong commutativity preserving on $R$ if $[f(x), f(y)]=[x, y]$ for all $x, y \in R$. In [16], Ma et al. described the structure of strong commutativity preserving generalized derivations on ideals and right ideals of prime rings. Ali et al. [4] studied strong commutativity preserving like derivations in rings with involution. Recently, Dar and Khan [12] proved the following: Let $R$ be a non-commutative 2-torsion free prime ring with involution of the second kind. If $R$ admits a generalized derivation $F: R \rightarrow R$ associated with a derivation $d: R \rightarrow R$ such that $\left[F(x), F\left(x^{*}\right)\right]-\left[x, x^{*}\right]=0$ for all $x \in R$, then $F(x)=x$ or $F(x)=-x$ for all $x \in R$.

Bell and Daif [6] showed that if $R$ is a prime ring admitting a non-zero derivation $d$ such that $d([x, y])=0$ for all $x, y \in R$, then $R$ is commutative. Ali et al. [3], studied the above mentioned result in the settings of prime rings with involution by replacing $y$ by $x^{*}$. Recently, Alahmadi et al. [2] obtained this result with generalized derivations of prime rings. After that, Idrissi and Oukhtite [14] considered this identity and proved the following: Let $R$ be a 2-torsion free prime ring with involution of the second kind. If $R$ admits a non-zero generalized derivation $F$ associated with a derivation $d$, 
then $R$ is commutative if and only if $F\left(\left[x, x^{*}\right]\right) \in Z(R)$ for all $x \in R$.

Very recently, Abbasi et al. [1] introduced the notion of skew Lie product in an involutive ring $R$ and examined a list of identities involving skew Lie product and left centralizers, by considering $R$ a prime ring. In this paper, we extend and unify several of their results to the class of generalized derivations.

\section{Preliminaries}

We shall frequently use the following basic commutator and anti-commutator identities:

$$
\begin{gathered}
{[u v, w]=u[v, w]+[u, w] v,[u, v w]=v[u, w]+[u, v] w} \\
(u v) \circ w=(u \circ w) v+u[v, w]=u(v \circ w)+[w, u] v, \\
u \circ(v w)=(u \circ v) w+v[w, u]=v(u \circ w)+[u, v] w
\end{gathered}
$$

which hold for all $u, v, w \in R$.

Let us bring some basic facts and extensions of several elementary results of this subject, which will be used throughout the proofs.

Lemma 1 [18, FACT 1] Let $R$ be a 2-torsion free prime ring with involution of the second kind. If $d(h)=0$ for all $h \in H(R) \cap Z(R)$, then $d$ vanishes on $Z(R)$.

Lemma 2 Let $R$ be a prime ring. For $a \in Z(R)$ and $b \in R$, if $a b \in Z(R)$, then either $b \in Z(R)$ or $a=0$.

Proof. Suppose that $a b \in Z(R)$. Then for each $x \in R, 0=[a b, x]=a[b, x]$. Taking $x u$ for $x$ in the last relation, we get

$$
0=a[b, x u]=a x[b, u]+a[b, x] u=a x[b, u], \quad x, u \in R .
$$

It can be seen that $a R[b, R]=(0)$. By using primness of $R$, we have either $a=0$ or $b \in Z(R)$, as desired.

Lemma 3 Let $R$ be a prime ring and $(0) \neq I$ be an ideal of $R$. If $x^{2} \in Z(R)$ for all $x \in I$, then $R$ is commutative.

Proof. From the given condition, we have $x^{2} \in Z(R)$ for all $x \in I$. By polarizing $x$, we obtain $[x \circ y, r]=0$ for all $x, y \in I, r \in R$. Replacing $y$ by $y r$, we have $y[[r, x], r]+[y, r][r, x]=0$. Replacing $y$ by $x y$ in the last expression and then using it, we obtain $[x, r] y[r, x]=0$ for all $x, y \in I$, $r \in R$. It implies that $[r, x] I[r, x]=(0)$ for all $x \in I, r \in R$, and hence, $I \subseteq Z(R)$. Thus, $R$ is commutative. 
Lemma 4 Let $R$ be a 2-torsion free prime ring with involution of the second kind and $(0) \neq I$ be $a *$-ideal of $R$. If $\nabla\left[x, x^{*}\right] \in Z(R)$ for all $x \in I$, then $R$ is commutative.

Proof. By the given hypothesis, $x x^{*}-x^{*} x^{*} \in Z(R)$ for all $x \in I$. Replacing $x$ by $x k_{c}$ where $0 \neq k_{c} \in S(R) \cap Z(R)$, we get that $x x^{*} \in Z(R)$. Polarizing we obtain $x y^{*}+y x^{*} \in Z(R)$. Replacing $x$ by $x k_{c}$, where $0 \neq k_{c} \in S(R) \cap Z(R)$, we get $\left(x y^{*}-y x^{*}\right) k_{c} \in Z(R)$. Since $0 \neq k_{c}$, by Lemma 2 , we get $x y^{*}-y x^{*} \in$ $Z(R)$. In this view, it follows that $2 x y^{*} \in Z(R)$ for all $x, y \in I$, which implies $x y^{*} \in Z(R)$. As $I$ is a $*$-ideal, we find $x y \in Z(R)$ for all $x, y \in I$. Thus, $I$ is commutative, and hence $R$ is commutative.

Lemma 5 Let $R$ be a 2-torsion free prime ring with involution of the second kind and $(0) \neq I$ be $a *$-ideal of $R$. If $\left[x, x^{*}\right] \in Z(R)$ for all $x \in I$, then $R$ is commutative.

Proof. Since $*$ is additive mapping, by using [9, Proposition 3.1], $\left[x, x^{*}\right]=0$. Replacing $x$ by $h+k$, where $h \in H(R) \cap I$ and $k \in S(R) \cap I$, we have

$$
h k=k h .
$$

Replacing $k$ by $x-x^{*}$ in (1), we get

$$
h\left(x-x^{*}\right)-\left(x-x^{*}\right) h=0, \quad x \in I, h \in H(R) \cap I .
$$

For $0 \neq k_{c} \in S(R) \cap Z(R)$ and $x \in I$, replacing $k$ by $k_{c}\left(x+x^{*}\right)$ in (1), we get $k_{c}\left(h\left(x+x^{*}\right)-\left(x+x^{*}\right) h\right)=0$. Using Lemma 2, we obtain

$$
h\left(x+x^{*}\right)-\left(x+x^{*}\right) h=0, \quad h \in H(R) \cap I .
$$

Adding (2) and (3) and then using torsion condition, we get

$$
h x=x h, \quad h \in H(R) \cap I .
$$

For any $y \in I$, replace $h$ by $y+y^{*}$ in (4) to obtain

$$
\left(y+y^{*}\right) x=x\left(y+y^{*}\right) .
$$

Let $0 \neq k_{c} \in S(R) \cap Z(R)$. Replacing $h$ by $k_{c}\left(y-y^{*}\right)$ in (4), we obtain $k_{c}\left(y-y^{*}\right) x=x k_{c}\left(y-y^{*}\right)$ for all $x, y \in I$. Using Lemma 2, we conclude

$$
\left(y-y^{*}\right) x=x\left(y-y^{*}\right) .
$$

Adding (5) and (6), we get $[x, y]=0$ for all $x, y \in I$. Hence, $R$ is commutative. 
Lemma 6 Let $R$ be a 2-torsion free prime ring with involution of second kind and $(0) \neq I$ be $a *$-ideal. If $x \circ x^{*} \in Z(R)$ for all $x \in I$, then $R$ is commutative.

Proof. Let us assume that $x \circ x^{*} \in Z(R)$ for all $x \in I$. We split the proof into the following two parts:

Case 1. Let $I \cap Z(R)=(0)$. Since $x \circ x^{*} \in I \cap Z(R)$, we have

$$
x \circ x^{*}=0, \quad x \in I .
$$

Polarizing (7), we obtain

$$
x \circ y^{*}+y \circ x^{*}=0, \quad x, y \in I .
$$

Replacing $y$ by $y k_{c}$ where $0 \neq k_{c} \in S(R) \cap Z(R)$, we conclude that

$$
-\left(x \circ y^{*}\right)+y \circ x^{*}=0, \quad x, y \in I .
$$

Adding (8) and (9), we get $y \circ x^{*}=0$ for all $x, y \in I$. It implies that $y \circ x=0$ for all $x, y \in I$. Replace $x$ by $x r$ where $r \in R$ to get $x[r, y]=0$. Thus, $R$ is commutative.

Case 2. Suppose that $I \cap Z(R) \neq(0)$. By our hypothesis, we have $x \circ x^{*} \in$ $Z(R)$ for all $x \in I$. Polarizing this expression, we get $x \circ y^{*}+y \circ x^{*} \in Z(R)$ for all $x, y \in I$. If we replace $y$ by $y k_{c}$ where $0 \neq k_{c} \in S(R) \cap Z(R)$, we find that $-x \circ y^{*}+y \circ x^{*} \in Z(R)$ for all $x, y \in I$. It follows that $y \circ x^{*} \in Z(R)$ for all $x, y \in I$. Now, replacing $y$ by $z$ where $0 \neq z \in I \cap Z(R)$, we get $2 x^{*} \in Z(R)$ for all $x \in I$. It implies that $I \subseteq Z(R)$, and hence, $R$ is commutative.

Lemma 7 Let $R$ be a 2-torsion free prime ring and $(0) \neq I$ be an ideal of $R$. If $R$ admits a nonzero generalized derivation $F$ with associated derivation $d$ such that $[F(x), y] \in Z(R)$ for all $x, y \in I$, then $R$ is commutative.

Proof. By the given hypothesis, $[F(x), y] \in Z(R)$ for all $x, y \in I$. Replacing $y$ by $y^{2}$ in given condition and using it, we conclude that $2[F(x), y] y \in$ $Z(R)$. It implies $y[F(x), y] \in Z(R)$, that is, for any $s \in R$, we have $s y[F(x), y]=y[F(x), y] s=y s[F(x), y]$. Thus, $[s, y][F(x), y]=0$ for all $x, y \in I, s \in R$. Replacing $s$ by $s r$ where $r \in R$, we have either $R$ is commutative or $[F(x), y]=0$ for all $x, y \in I$. Replace $x$ by $x y$ in the latter case to obtain $[x, y] d(y)+x[d(y), y]=0$ for all $x, y \in I$. Thereby, replacing $x$ by $r x$ where $r \in R$, we have $[r, y] x d(y)=0$ for all $x, y \in I, r \in R$. It implies that for each $y \in I$, either $[R, y]=(0)$ or $d(y)=0$. Then, $I$ is the set theocratic union of the additive subgroups $M=\{y \in I:[R, y]=(0)\}$ and $N=\{y \in I: d(y)=0\}$, which is not possible. Therefore, either $I=M$ or $I=N$. In other words, we have either $I \subseteq Z(R)$, and hence, $R$ is commutative, or $d(x)=0$ for all $x \in I$. Let us consider the case $d(x)=0$ for all 
$x \in I$. It implies that $0=d(x r)=x d(r)$ for all $x \in I$ and $r \in R$. Since $R$ is prime and $I$ is a non-zero ideal of $R$, we get $d=0$.

Now, replace $x$ by $x t$ with $t \in R$ in $[F(x), y]=0$ to obtain $F(I)[R, I]=$ (0). In view of primeness of $R$, it follows that either $R$ is commutative or $F(I)=(0)$. It is not difficult to see that the situation $F(I)=(0)$ implies $F=0$, which is a contradiction. Hence, $R$ is commutative.

Lemma 8 Let $R$ be a 2-torsion free prime ring and $(0) \neq I$ be an ideal of $R$. If there exists $0 \neq a \in R$ such that $[x, y] a \in Z(R)$ for all $x, y \in I$, then $R$ is commutative.

Proof. Let us assume that there exists $a \in R, a \neq 0$, such that $[x, y] a \in$ $Z(R)$ for all $x, y \in I$, that is, $[[x, y] a, r]=0$ for all $y \in I, r \in R$. Then

$$
[x, y][a, r]+[[x, y], r] a=0, \quad y \in I, r \in R .
$$

Replacing $x$ by $y x$ in $(10)$, we obtain

$$
y[x, y][a, r]+y[[x, y], r] a+[y, r][x, y] a=0 .
$$

Using (10) and (11), we compute $[y, r][x, y] a=0$. Replacing $r$ by $s r$ where $s \in R$, we obtain $[y, s] R[x, y] a=(0)$ for all $x, y \in I$ and $s \in R$. Now it is straight forward to conclude that $R$ is commutative as $a \neq 0$.

Lemma 9 Let $R$ be a 2-torsion free prime ring and $(0) \neq I$ be an ideal. If there exists $0 \neq a \in R$ such that $a[x, y] \in Z(R)$ for all $x, y \in I$, then $R$ is commutative.

The proof is similar to that of Lemma 8 .

Lemma 10 [17, THEOREM 2] Let $R$ be a 2-torsion free prime ring with involution $*, F$ be a non-zero generalized derivation associated with derivation $d$ and $I$ be a non-zero $*$-ideal. If $F(x \circ y) \in Z(R)$ for all $x, y \in I$, then $R$ is commutative.

Lemma 11 [19, THEOREM 1] Let $R$ be a 2-torsion free prime ring with involution $*, d$ be a non-zero derivation and $I$ be a non-zero $*$-ideal. If $[d(x), x] \in Z(R)$ for all $x \in I$, then $R$ is commutative.

Lemma 12 [15, TheOREM 3] Let $R$ be a left faithful ring. Then every generalized derivation $F$ on a dense right ideal of $R$ can be extended to the right Utumi quotient ring $U$ of $R$ and assumes the form $F(x)=a x+\delta(x)$ for some $a \in U$ and a derivation $\delta$ on $U$. 


\section{Main results}

Theorem 1 Let $R$ be a 2-torsion free prime ring with involution of the second kind and $(0) \neq I$ be $a *$-ideal of $R$. If $F$ and $G$ are generalized derivations of $R$ with associated derivations $d$ and $\delta$, respectively, then $R$ is commutative if and only if $\left[F(x), G\left(x^{*}\right)\right] \pm \nabla\left[x, x^{*}\right] \in Z(R)$ for all $x \in I$.

Proof. The non-trivial implication is to show that $\left[F(x), G\left(x^{*}\right)\right] \pm \nabla\left[x, x^{*}\right] \in$ $Z(R)$ for all $x \in I$ implies commutativity of $R$. If either $F=0$ or $G=0$, then the conclusion follows from Lemma 4. Therefore, both $F$ and $G$ are non-zero onwards.

Let us assume that

$$
\left[F(x), G\left(x^{*}\right)\right]+\nabla\left[x, x^{*}\right] \in Z(R), \quad x \in I .
$$

Polarizing (12), we obtain

$$
\left[F(x), G\left(y^{*}\right)\right]+\left[F(y), G\left(x^{*}\right)\right]+\nabla\left[x, y^{*}\right]+\nabla\left[y, x^{*}\right] \in Z(R), \quad x, y \in I .
$$

For any $h_{c} \in H(R) \cap Z(R)$, substituting $y h_{c}$ for $y$ in $(13)$, we get

$$
\begin{array}{r}
{\left[F(x), G\left(y^{*}\right) h_{c}+y^{*} \delta\left(h_{c}\right)\right]+\left[F(y) h_{c}+y d\left(h_{c}\right), G\left(x^{*}\right)\right]+} \\
\nabla\left[x, y^{*}\right] h_{c}+\nabla\left[y, x^{*}\right] h_{c} \in Z(R) .
\end{array}
$$

Using (13) in 14), we find

$$
\left[F(x), y^{*}\right] \delta\left(h_{c}\right)+\left[y, G\left(x^{*}\right)\right] d\left(h_{c}\right) \in Z(R), \quad x, y \in I .
$$

Take $0 \neq k_{c} \in S(R) \cap Z(R)$ and replace $y$ by $y k_{c}$ in 15 to obtain

$$
-\left[F(x), y^{*}\right] k_{c} \delta\left(h_{c}\right)+\left[y, G\left(x^{*}\right)\right] k_{c} d\left(h_{c}\right) \in Z(R) .
$$

It implies that

$$
-\left[F(x), y^{*}\right] \delta\left(h_{c}\right)+\left[y, G\left(x^{*}\right)\right] d\left(h_{c}\right) \in Z(R)
$$

for all $x, y \in I$ and $h_{c} \in H(R) \cap Z(R)$. Adding (15) and (16), we get $\left[y, G\left(x^{*}\right)\right] d\left(h_{c}\right) \in Z(R)$. It implies that either $\left[y, G\left(x^{*}\right)\right] \in Z(R)$ for all $x, y \in I$ or $d\left(h_{c}\right)=0$ for all $h_{c} \in H(R) \cap Z(R)$. In the former case, $R$ is commutative by Lemma 7. In the latter case, we have $d\left(h_{c}\right)=0$, and by Lemma 1, we get $d(Z(R))=(0)$. Using this in (15) and then applying the similar arguments, we obtain $\delta(Z(R))=(0)$. Replace $y$ by $y k_{c}$ where $0 \neq k_{c} \in S(R) \cap Z(R)$ in (13) to get

$$
-\left[F(x), G\left(y^{*}\right)\right] k_{c}+\left[F(y), G\left(x^{*}\right)\right] k_{c}-\nabla\left[x, y^{*}\right] k_{c}+y x^{*} k_{c}+y^{*} x^{*} k_{c} \in Z(R) .
$$


It implies

$$
-\left[F(x), G\left(y^{*}\right)\right]+\left[F(y), G\left(x^{*}\right)\right]-\nabla\left[x, y^{*}\right]+y x^{*}+y^{*} x^{*} \in Z(R)
$$

for all $x, y \in I$. Adding (13) and (17), we get

$$
2\left[F(y), G\left(x^{*}\right)\right]+2 y x^{*} \in Z(R), \quad x, y \in I .
$$

Replacing $y$ by $x$ in (18) and thereby comparing it with (12), we obtain $\left(x^{*}\right)^{2} \in Z(R)$. By Lemma 3, $R$ is commutative.

By taking $G=-G$ in the above proof, we get the same conclusion from $\left[F(x), G\left(x^{*}\right)\right]-\nabla\left[x, x^{*}\right] \in Z(R)$ for all $x \in I$. This completes the proof.

Corollary 1 Let $R$ be a 2-torsion free prime ring with involution of the second kind and $(0) \neq I$ be $a *$-ideal of $R$. If $G$ is a generalized derivation of $R$ with associated derivation $\delta$, then $R$ is commutative if and only if $\left[x, G\left(x^{*}\right)\right] \pm \nabla\left[x, x^{*}\right] \in Z(R)$ for all $x \in I$.

Corollary 2 Let $R$ be a 2-torsion free prime ring with involution of the second kind and $(0) \neq I$ be $a *$-ideal of $R$. If $F$ is a generalized derivation of $R$ with associated derivation $d$, then $R$ is commutative if and only if $\left[F(x), x^{*}\right] \pm \nabla\left[x, x^{*}\right] \in Z(R)$ for all $x \in I$.

Proposition 1 Let $R$ be a 2-torsion free non-commutative prime ring and $(0) \neq I$ be an ideal of $R$. If $G$ is a generalized derivation of $R$ with associated derivation $\delta$ such that $G(x) x \in Z(R)$ for all $x \in I$, then $G=0$.

Proof. Let us assume that $G(x) x \in Z(R)$ for all $x \in I$. By polarizing, we obtain

$$
G(x) y+G(y) x \in Z(R) .
$$

Note that $G(x) y+G(y) x \in I$ for all $x, y \in I$. Thus, we divide the proof into the following two parts:

Case 1. Let $I \cap Z(R)=(0)$. Then we have

$$
G(x) y+G(y) x=0, \quad x, y \in I .
$$

Replacing $y$ by $y r$ where $r \in R$, we can write

$$
G(x) y r+G(y) r x+y \delta(r) x=0 .
$$

From 20, and (21), we obtain

$$
G(y)[r, x]+y \delta(r) x=0, \quad x, y \in I, r \in R .
$$

Replacing $x$ by xs in (22) and comparing the obtained relation with (22), we compute $G(y) x[r, s]=0$ for all $x, y \in I$ and $r, s \in R$. It implies that 
$G(y)=0$ for all $y \in I$. Replacing $y$ by $y r$ in the obtained expression where $r \in R$, we get $y \delta(r)=0$. It forces that $\delta=0$. Thus, for any $x \in R$ and $y \in I$, we have $0=G(x y)=G(x) y$, that is, $G(R) I=(0)$. It follows that $G=0$.

Case 2. Assume that $I \cap Z(R) \neq(0)$. Replace $y$ and $x$ by $z$ where $0 \neq$ $z \in I \cap Z(R)$ in $(19)$ to get $G(z) z \in Z(R)$. It implies that for each $z \in$ $I \cap Z(R)$, either $G(z) \in Z(R)$ or $z=0$. Therefore, $G(z) \in Z(R)$ for all $z \in I \cap Z(R)$. Replace $y$ by $z$ where $0 \neq z \in I \cap Z(R)$ in (19) to obtain $G(x) z+G(z) x \in Z(R)$. Commuting with $x$ and using the fact that $G(z) \in Z(R)$, we get $[G(x), x] z=0$ for all $x \in I$. By Lemma 2, we have $[G(x), x]=0$ for all $x \in I$. Replacing $x$ by $z x$, we have $[\delta(x), x]=0$ for all $x \in I$. According to Lemma 11, it implies $\delta=0$. By Lemma 12, we have $G(x)=a x$. In particular, taking $x=z$ where $0 \neq z \in I \cap Z(R)$, we conclude that $a \in Z(R)$. From the initial hypothesis, we find $a x^{2} \in Z(R)$ for all $x \in I$. It implies that either $x^{2} \in Z(R)$ or $a=0$. By Lemma 3, it follows that the former case yields a contradiction, thus, we conclude that $a=0$, and hence, $G=0$.

The following result extends Theorem 10 of [1].

Theorem 2 Let $R$ be a 2-torsion free non-commutative prime ring with involution of the second kind and $(0) \neq I$ be $a *$-ideal of $R$. If $F$ and $G$ are generalized derivations with associated derivations $d$ and $\delta$, respectively, such that $F\left(x \circ x^{*}\right) \pm \nabla\left[x, G\left(x^{*}\right)\right] \in Z(R)$ for all $x \in I$, then $F=G=0$.

Proof. Let us assume that

$$
F\left(x \circ x^{*}\right)+\nabla\left[x, G\left(x^{*}\right)\right] \in Z(R), \quad x \in I .
$$

Polarize 23) to get

$$
F\left(x \circ y^{*}\right)+F\left(y \circ x^{*}\right)+\nabla\left[x, G\left(y^{*}\right)\right]+\nabla\left[y, G\left(x^{*}\right)\right] \in Z(R), \quad x, y \in I .
$$

Replacing $y$ by $y h_{c}$ in (24) where $h_{c} \in H(R) \cap Z(R)$, we get

$$
\begin{aligned}
& F\left(x \circ y^{*}\right) h_{c}+\left(x \circ y^{*}\right) d\left(h_{c}\right)+F\left(y \circ x^{*}\right) h_{c}+\left(y \circ x^{*}\right) d\left(h_{c}\right) \\
& +\nabla\left[x, G\left(y^{*}\right)\right] h_{c}+\nabla\left[x, y^{*}\right] \delta\left(h_{c}\right)+\nabla\left[y, G\left(x^{*}\right)\right] h_{c} \in Z(R) .
\end{aligned}
$$

Using 24 in 25, we can write

$$
\left(x \circ y^{*}\right) d\left(h_{c}\right)+\left(y \circ x^{*}\right) d\left(h_{c}\right)+x y^{*} \delta\left(h_{c}\right)-y^{*} x^{*} \delta\left(h_{c}\right) \in Z(R) .
$$

Replacing $y$ by $y k_{c}$ in 26 where $0 \neq k_{c} \in S(R) \cap Z(R)$, we obtain

$$
-\left(x \circ y^{*}\right) d\left(h_{c}\right)+\left(y \circ x^{*}\right) d\left(h_{c}\right)-x y^{*} \delta\left(h_{c}\right)+y^{*} x^{*} \delta\left(h_{c}\right) \in Z(R) .
$$


From (26) and (27), it follows that $\left(y \circ x^{*}\right) d\left(h_{c}\right) \in Z(R)$. It implies that either $\left(y \circ x^{*}\right) \in Z(R)$ for all $x, y \in I$ or $d\left(h_{c}\right)=0$ for all $h_{c} \in H(R) \cap Z(R)$. In the former case, $R$ is commutative by Lemma 6 , which is a contradiction. Thus, we have $d\left(h_{c}\right)=0$. By Lemma 1, we have $d(Z(R))=(0)$. From (26), we obtain

$$
x y^{*} \delta\left(h_{c}\right)-y^{*} x^{*} \delta\left(h_{c}\right) \in Z(R) .
$$

Now by replacing $x$ by $x k_{c}$ in $(28)$ where $0 \neq k_{c} \in S(R) \cap Z(R)$, we find that $x y^{*} \delta\left(h_{c}\right)+y^{*} x^{*} \delta\left(h_{c}\right) \in Z(R)$ for all $x, y \in I$. Combining this relation with (28), we get $x y^{*} \delta\left(h_{c}\right) \in Z(R)$ for all $x, y \in I$. In view of Lemma 2 , it follows that either $x y^{*} \in Z(R)$ for all $x, y \in I$ or $\delta\left(h_{c}\right)=0$ for all $h_{c} \in$ $H(R) \cap Z(R)$. In the former case, $R$ is commutative by Lemma 3 , which is a contradiction. Therefore, we have $\delta\left(h_{c}\right)=0$, which implies $\delta(Z(R))=(0)$ by Lemma 1. Replace $y$ by $y k_{c}$ where $0 \neq k_{c} \in S(R) \cap Z(R)$ in (24) to obtain

$-F\left(x \circ y^{*}\right) k_{c}+F\left(y \circ x^{*}\right) k_{c}-\nabla\left[x, G\left(y^{*}\right)\right] k_{c}+y G\left(x^{*}\right) k_{c}+G\left(x^{*}\right) y^{*} k_{c} \in Z(R)$.

It implies that for all $x, y \in I$,

$$
-F\left(x \circ y^{*}\right)+F\left(y \circ x^{*}\right)-\nabla\left[x, G\left(y^{*}\right)\right]+y G\left(x^{*}\right)+G\left(x^{*}\right) y^{*} \in Z(R) .
$$

Comparing (24) and (29), we get $2 F\left(y \circ x^{*}\right)+2 y G\left(x^{*}\right) \in Z(R)$ for all $x, y \in I$. Hence,

$$
F\left(y \circ x^{*}\right)+y G\left(x^{*}\right) \in Z(R), \quad x, y \in I .
$$

Replacing $y$ by $x$ in (30) and comparing the obtained expression with 23), we find $G\left(x^{*}\right) x^{*} \in Z(R)$ for all $x \in I$. By Proposition 1 , we get $G=0$. Thereby, using Lemma 10 in $(30)$, we obtain $F=0$.

By taking $G=-G$ in the above proof, we get the same conclusion from $F\left(x \circ x^{*}\right)-\nabla\left[x, G\left(x^{*}\right)\right] \in Z(R)$ for all $x \in I$. This completes the proof.

Corollary 3 Let $R$ be a 2-torsion free prime ring with involution of the second kind. If $F$ is a generalized derivation with associated derivation $d$ of $R$ such that $F\left(x \circ x^{*}\right) \pm \nabla\left[x, x^{*}\right] \in Z(R)$ for all $x \in R$, then $R$ is commutative.

Proof. Assume that $R$ is non commutative. With $G(x)=x$ for all $x \in R$ and $I=R$, the present situation becomes a particular case of Theorem 2, a fortiori, $F=G=0$, which is a contradiction. Hence, $R$ is commutative.

The following corollary from Theorem 1 is a generalization of [1, Theorem 1].

Corollary 4 Let $R$ be a 2-torsion free prime ring with involution of the second kind and $(0) \neq I$ be $a *$-ideal of $R$. If $G$ is a non-zero generalized derivation with associated derivation $\delta$ of $R$ such that $\nabla\left[x, G\left(x^{*}\right)\right] \in Z(R)$ for all $x \in I$, then $R$ is commutative. 
We now give a generalization of [1, Theorem 11].

Theorem 3 Let $R$ be a 2-torsion free non-commutative prime ring with involution of second kind and $(0) \neq I$ be $a *$-ideal of $R$. If $F$ and $G$ are generalized derivations with associated derivations $d$ and $\delta$, respectively, such that $F\left(\nabla\left[x, x^{*}\right]\right) \pm \nabla\left[x, G\left(x^{*}\right)\right] \in Z(R)$ for all $x \in I$, then there exists $\lambda \in C$ such that $F(x)=\mp \lambda x$ and $G(x)=\lambda x$ for all $x \in R$.

Proof. Let us assume that

$$
F\left(\nabla\left[x, x^{*}\right]\right)+\nabla\left[x, G\left(x^{*}\right)\right] \in Z(R), \quad x \in I .
$$

Polarizing (31), for all $x, y \in I$, we can write

$$
F\left(\nabla\left[x, y^{*}\right]\right)+F\left(\nabla\left[y, x^{*}\right]\right)+\nabla\left[x, G\left(y^{*}\right)\right]+\nabla\left[y, G\left(x^{*}\right)\right] \in Z(R) .
$$

Replace $y$ by $y h_{c}$ with $h_{c} \in H(R) \cap Z(R)$ in (32) to obtain

$$
\begin{array}{r}
F\left(\nabla\left[x, y^{*}\right]\right) h_{c}+\nabla\left[x, y^{*}\right] d\left(h_{c}\right)+F\left(\nabla\left[y, x^{*}\right]\right) h_{c}+\nabla\left[y, x^{*}\right] d\left(h_{c}\right) \\
+\nabla\left[x, G\left(y^{*}\right)\right] h_{c}+\nabla\left[x, y^{*}\right] \delta\left(h_{c}\right)+\nabla\left[y, G\left(x^{*}\right)\right] h_{c} \in Z(R) .
\end{array}
$$

By comparing (32) and (33), we conclude that

$$
\nabla\left[x, y^{*}\right] d\left(h_{c}\right)+\nabla\left[y, x^{*}\right] d\left(h_{c}\right)+\nabla\left[x, y^{*}\right] \delta\left(h_{c}\right) \in Z(R)
$$

for all $x, y \in I$ and $h_{c} \in H(R) \cap Z(R)$. Replace $y$ by $y k_{c}$ where $0 \neq k_{c} \in$ $S(R) \cap Z(R)$ in (34) to get

$$
\left(-x y^{*}+y^{*} x^{*}\right) k_{c} d\left(h_{c}\right)+\left(y x^{*}+x^{*} y^{*}\right) k_{c} d\left(h_{c}\right)+\left(-x y^{*}+y^{*} x^{*}\right) k_{c} \delta\left(h_{c}\right) \in Z(R) .
$$

From Lemma 2, it follows that

$$
\left(-x y^{*}+y^{*} x^{*}\right) d\left(h_{c}\right)+\left(y x^{*}+x^{*} y^{*}\right) d\left(h_{c}\right)+\left(-x y^{*}+y^{*} x^{*}\right) \delta\left(h_{c}\right) \in Z(R) .
$$

By adding (34) and (35), we obtain

$$
2 y x^{*} d\left(h_{c}\right) \in Z(R), \quad x, y \in I .
$$

According to Lemma 2, we have either $y x^{*} \in Z(R)$ for all $x, y \in I$ or $d\left(h_{c}\right)=0$ for all $h_{c} \in H(R)$. In former case, $R$ is commutative by Lemma 3 , which is a contradiction. Thus, we have $d\left(h_{c}\right)=0$ for all $h_{c} \in H(R) \cap Z(R)$ and by Lemma1, $d(Z(R))=(0)$. From (34), we obtain $\nabla\left[y, x^{*}\right] \delta\left(h_{c}\right) \in Z(R)$ for all $x, y \in I$ and $h_{c} \in H(R) \cap Z(R)$. It implies that either $\nabla\left[y, x^{*}\right] \in Z(R)$ or $\delta\left(h_{c}\right)=0$. In former case, a contradiction follows by Lemma 4 . Therefore, we have $\delta\left(h_{c}\right)=0$, which implies $\delta(Z(R))=(0)$. 
Replace $y$ by $y k_{c}$ with $0 \neq k_{c} \in S(R) \cap Z(R)$ in $(32)$ to obtain

$$
\begin{array}{r}
F\left(-x y^{*}+y^{*} x^{*}\right) k_{c}+F\left(y x^{*}+x^{*} y^{*}\right) k_{c}+\left(-x G\left(y^{*}\right)+G\left(y^{*}\right) x^{*}\right) k_{c}+ \\
\left(y G\left(x^{*}\right)+G\left(x^{*}\right) y^{*}\right) k_{c} \in Z(R) .
\end{array}
$$

It implies that for all $x, y \in I$ and $h_{c} \in H(R) \cap Z(R)$,

$$
\begin{array}{r}
F\left(-x y^{*}+y^{*} x^{*}\right)+F\left(y x^{*}+x^{*} y^{*}\right)+\left(-x G\left(y^{*}\right)+G\left(y^{*}\right) x^{*}\right)+ \\
\left(y G\left(x^{*}\right)+G\left(x^{*}\right) y^{*}\right) \in Z(R) .
\end{array}
$$

Adding (32) and (36), we get $F\left(y x^{*}\right)+y G\left(x^{*}\right) \in Z(R)$. Therefore,

$$
F(y) x+y d(x)+y G(x) \in Z(R), \quad x, y \in I .
$$

Case 1. Assume that $I \cap Z(R)=(0)$. Since $F(y) x+y d(x)+y G(x) \in I$ as well as $F(y) x+y d(x)+y G(x) \in Z(R)$ for all $x, y \in I$, we find

$$
F(y) x+y d(x)+y G(x)=0, \quad x, y \in I .
$$

Replacing $x$ by $x r$ in (38) where $r \in R$, we get $y x d(r)+y x \delta(r)=0$. It implies $(d+\delta)(x)=0$ for all $x \in R$. Using Lemma 12 in (38), we compute $F(y) x+y d(x)+y b x+y \delta(x)=0$. It implies $F(y)=-y b$ for all $y \in I$. Using Lemma 12 once again, we obtain $d(y)=-y b-a y$. Replacing $x$ by $x y$, we get $d(x y)=-x y b-a x y=d(x) y+x d(y)$, which gives $-x b y-a x y-x y b-x a y=$ $-x y b-a x y$. It implies $x(a+b) y=0$ for all $x, y \in I$. Thus, $a=-b$. From (38), we obtain $-y b x-y x b+y b x+y G(x)=0$. It implies $G(x)=x b$ for all $x \in I$. Using it in (31), we get $-\nabla\left[x, x^{*}\right] b+\nabla\left[x, x^{*} b\right] \in Z(R)$, which implies $x^{*}\left[x^{*}, b\right] \in Z(R)$ for all $x \in I$. Replacing $x$ by $x^{*}$ in the last relation, we get $x[x, b] \in Z(R)$ for all $x \in I$. Also, for each $x \in I, x[x, b] \in I$. Since $I \cap Z(R)=(0)$, we have $x[x, b]=0$. Polarizing above equation, we obtain

$$
y[x, b]+x[y, b]=0, \quad x, y \in I .
$$

Replacing $y$ by $r y$ with $r \in R$, we can write

$$
r y[x, b]+x r[y, b]+x[r, b] y=0, \quad x, y \in I, r \in R .
$$

Comparing (39) and (40), we obtain

$$
[x, r][y, b]+x[r, b] y=0 .
$$

Replacing $x$ by $s x$ with $s \in R$ in (41) and comparing the obtained relation with (41), we compute $[r, s] x[y, b]=0$ for all $x, y \in I, r, s \in R$. It implies $b \in C$. Thus, $F(x)=-b x$ and $G(x)=b x$ for all $x \in R$. Therefore, we get the desired result.

Case 2. Let $I \cap Z(R) \neq(0)$. Then, replacing $x$ by $z$ where $0 \neq z \in$ 
$Z(R)$ in (37), we obtain $F(y) z+y G(z) \in Z(R)$. By Lemma 12 , we have $F(y) z+y b z \in Z(R)$ for all $y \in I$. By Lemma 2 , we find

$$
F(y)+y b \in Z(R), \quad y \in I .
$$

From (37), we get

$$
F(y) x+y d(x)+y b x+y \delta(x) \in Z(R), \quad x, y \in I .
$$

Commuting with $x$ and using (42) and (43), we get $[y d(x)+y \delta(x), x]=0$ for all $x, y \in I$. It implies $y[d(x)+\delta(x), x]+[y, x](d(x)+\delta(x))=0$ for all $x, y \in I$. Replacing $y$ by sy where $s \in R$, we find

$$
d(x)=-\delta(x), \quad x \in I .
$$

Using (44) in (43), we obtain $(F(y)+y b) x \in Z(R)$ for all $x, y \in I$. From 42) and Lemma 2 , we have $F(y)=-y b$ or $I \subseteq Z(R)$. The latter case leads to a contradiction. Therefore, we have $F(y)=-y b$ for all $y \in I$. Using Lemma 12, we have $a y+d(y)=-y b$. In particular, for $y \in Z(R)$, we get $a=-b$. Thus, we have $d(y)=b y-y b$. By (44), we have $\delta(y)=-b y+y b$. Also, we have $G(y)=b y+\delta(y)$. It implies $G(y)=y b$ for all $y \in R$. From (31), we obtain $-\nabla\left[x, x^{*}\right] b+\nabla\left[x, x^{*} b\right] \in Z(R)$, which implies $x^{*}\left[x^{*}, b\right] \in Z(R)$. Replacing $x$ by $x^{*}$, we have $x[x, b] \in Z(R)$ for all $x \in I$. Polarize above equation to obtain

$$
y[x, b]+x[y, b] \in Z(R), \quad x, y \in I .
$$

In particular, if we take $0 \neq y \in I \cap Z(R)$ and use Lemma 2, we get $[x, b] \in Z(R)$ for all $x \in I$. Thus, $b \in Z(R)$. Therefore, we have $F(x)=-b x$ and $G(x)=b x$ for all $x \in R$.

By taking $G=-G$ in the above proof, we get the same conclusion from $F\left(\nabla\left[x, x^{*}\right]\right)-\nabla\left[x, G\left(x^{*}\right)\right] \in Z(R)$ for all $x \in I$. This completes the proof.

Corollary 5 Let $R$ be a 2-torsion free non-commutative prime ring with involution of second kind. If $G$ is a generalized derivation of $R$ with associated derivation $\delta$ such that $\left(\nabla\left[x, x^{*}\right]\right) \pm \nabla\left[x, G\left(x^{*}\right)\right] \in Z(R)$ for all $x \in I$, then $G(x)=\mp x$ for all $x \in R$.

The following result is a generalization of [1, Theorem 2].

Corollary 6 Let $R$ be a 2-torsion free prime ring with involution of second kind and $(0) \neq I$ be $a *$-ideal of $R$. If $F$ is a non-zero generalized derivation of $R$ with associated derivation $d$ such that $F\left(\nabla\left[x, x^{*}\right]\right) \in Z(R)$ for all $x \in I$, then $R$ is commutative. 
Theorem 4 Let $R$ be a 2-torsion free non-commutative prime ring with involution of the second kind and $(0) \neq I$ be $a *$-ideal of $R$. If $F$ and $G$ are generalized derivations of $R$ with associated derivations $d$ and $\delta$, respectively, such that $F(x) \circ x^{*} \pm \nabla\left[x, G\left(x^{*}\right)\right] \in Z(R)$ for all $x \in I$, then $F=G=0$.

Proof. Let us assume that

$$
F(x) \circ x^{*}+\nabla\left[x, G\left(x^{*}\right)\right] \in Z(R), \quad x \in I .
$$

Polarize (45) to obtain

$$
F(x) \circ y^{*}+F(y) \circ x^{*}+\nabla\left[x, G\left(y^{*}\right)\right]+\nabla\left[y, G\left(x^{*}\right)\right] \in Z(R), \quad x, y \in I .
$$

Replacing $y$ by $y h_{c}$ with $h_{c} \in H(R) \cap Z(R)$ in (46), we obtain

$$
\begin{array}{r}
\left(F(x) \circ y^{*}\right) h_{c}+\left(F(y) \circ x^{*}\right) h_{c}+\left(y \circ x^{*}\right) d\left(h_{c}\right)+\nabla\left[x, G\left(y^{*}\right)\right] h_{c} \\
+\nabla\left[x, y^{*}\right] \delta\left(h_{c}\right)+\nabla\left[y, G\left(x^{*}\right)\right] h_{c} \in Z(R) .
\end{array}
$$

Comparing (46) and (47), we get

$$
\left(y \circ x^{*}\right) d\left(h_{c}\right)+\nabla\left[x, y^{*}\right] \delta\left(h_{c}\right) \in Z(R),
$$

$x, y \in I, h_{c} \in H(R) \cap Z(R)$. Replacing $y$ by $y k_{c}$ where $0 \neq k_{c} \in S(R) \cap Z(R)$, we obtain $\left(y \circ x^{*}\right) k_{c} d\left(h_{c}\right)+\left(-x y^{*}+y^{*} x^{*}\right) k_{c} \delta\left(h_{c}\right) \in Z(R)$. It implies that for all $x, y \in I$ and $h_{c} \in H(R) \cap Z(R)$,

$$
\left(y \circ x^{*}\right) d\left(h_{c}\right)+\left(-x y^{*}+y^{*} x^{*}\right) \delta\left(h_{c}\right) \in Z(R) .
$$

Adding (48) and 49), we obtain

$$
\left(y \circ x^{*}\right) d\left(h_{c}\right) \in Z(R) .
$$

Lemma 2 yields that either $x \circ y \in Z(R)$ for all $x, y \in I$ or $d\left(h_{c}\right)=0$ for all $h_{c} \in H(R) \cap Z(R)$. The former case leads to a contradiction by Lemma 6 . Thus, we have $d\left(h_{c}\right)=0$, which implies $d(Z(R))=(0)$. From 449$)$, we obtain $\nabla\left[x, y^{*}\right] \delta\left(h_{c}\right) \in Z(R)$ for all $x, y \in I, h_{c} \in H(R) \cap Z(R)$. Lemma 2 implies either $\nabla\left[x, y^{*}\right] \in Z(R)$ or $\delta\left(h_{c}\right)=0$. In the former case, a contradiction follows from Lemma 4. Therefore, we have $\delta\left(h_{c}\right)=0$, and then by Lemma 1. $\delta(Z(R))=(0)$. Replacing $y$ by $y k_{c}$ where $0 \neq k_{c} \in S(R) \cap Z(R)$ in (46), we obtain

$$
\begin{array}{r}
-\left(F(x) \circ y^{*}\right) k_{c}+\left(F(y) \circ x^{*}\right) k_{c}+\left(-x G\left(y^{*}\right)\right. \\
\left.+G\left(y^{*}\right) x^{*}\right) k_{c}+\left(y G\left(x^{*}\right)+G\left(x^{*}\right) y^{*}\right) k_{c} \in Z(R) .
\end{array}
$$

It implies

$$
\begin{array}{r}
-F(x) \circ y^{*}+F(y) \circ x^{*}-x G\left(y^{*}\right)+G\left(y^{*}\right) x^{*} \\
+y G\left(x^{*}\right)+G\left(x^{*}\right) y^{*} \in Z(R)
\end{array}
$$


for all $x, y \in I$ and $h_{c} \in H(R) \cap Z(R)$. Combining (46) and (50), we obtain $2 F(y) \circ x^{*}+2 y G\left(x^{*}\right) \in Z(R)$ for all $x, y \in I$, which implies

$$
F(y) \circ x+y G(x) \in Z(R), \quad x, y \in I .
$$

Case 1. If $I \cap Z(R)=(0)$, then

$$
F(y) \circ x+y G(x)=0, \quad x, y \in I .
$$

Replacing $x$ by $x r$ in (52), where $r \in R$, we have

$$
(F(y) \circ x) r+x[r, F(y)]+y G(x) r+y x \delta(r)=0, \quad x, y \in I, r \in R .
$$

Comparing (52) and (53), we obtain

$$
x[r, F(y)]+y x \delta(r)=0 .
$$

Replacing $x$ by $s x$ where $s \in R$ in (54), we have

$$
s x[r, F(y)]+y s x \delta(r)=0 .
$$

Comparing (54) and (55), we obtain $[y, s] x \delta(r)=0$ for all $x, y \in I, r \in R$, which implies $\delta=0$. Using it in (54), we get $x[r, F(y)]=0$ for all $x, y \in I$ and $r \in R$. In particular, we have $x[z, F(y)]=0$ for all $x, y, z \in I$. Using primeness of $I$, we get $[z, F(y)]=0$ for all $y, z \in I$, and hence, the result follows from Lemma 7 .

Case 2. Let us suppose that $I \cap Z(R) \neq(0)$. Then we replace $x$ by $z$ where $0 \neq z \in I \cap Z(R)$ in (51) to obtain $2 F(y) z+y G(z) \in Z(R)$. In view of Lemma 12 , it follows that

$$
2 F(y)+y b \in Z(R), \quad y \in I .
$$

Replacing $y$ by $y r$ in (56)), where $r \in R$, we find $2 F(y) r+2 y d(r)+y r b \in$ $Z(R)$ for all $x, y \in I, r \in R$, which implies $(2 F(y)+y b) r+y[r, b]+2 y d(r) \in$ $Z(R)$. From (56), we compute $[y[r, b]+2 y d(r), r]=0$, which implies

$$
y[[r, b]+2 d(r), r]+[y, r]([r, b]+2 d(r))=0, \quad y \in I, r \in R .
$$

Replace $y$ by sy, where $s \in R$, in the above relation and then compare it with (57) to get $[s, r] y([r, b]+2 d(r))=0$ for all $y \in I, r \in R$. It implies $2 d(r)=[b, r]$, applying which with Lemma 12 in (56), we obtain $2 a y+b y-$ $y b+y b \in Z(R)$ for all $y \in I$. Thus, $2 a=-b$ and hence $2 F(y)=2 a y+[b, y]=$ $-y b$. Using it in (51), we compute $-y b x-x y b+2 y b x+2 y \delta(x) \in Z(R)$ for all $x, y \in I$. In particular, taking $z$ instead of $y$ in the last expression, we compute $(2 \delta(x)+[b, x]) z \in Z(R)$. Since $z \neq 0$, by Lemma 2 , we have $2 \delta(x)=[x, b]$ for all $x \in I$. It implies $2 G(x)=2 b x+x b-b x=b x+x b$. 
From (51), we get $2 F(y) \circ x+y 2 G(x) \in Z(R)$ for all $x, y \in I$. In view of the above computations, it implies that $(-y b) \circ x+y(b x+x b) \in Z(R)$ for all $x, y \in I$. On simplifying it, we get $[y, x] b \in Z(R)$ for all $x, y \in I$. By Lemma 8, we obtain $b=0$, which implies $F=G=0$, as desired.

By taking $G=-G$ in the above proof, we get the same conclusion from $F(x) \circ x^{*}-\nabla\left[x, G\left(x^{*}\right)\right] \in Z(R)$ for all $x \in I$. This completes the proof.

The following statement is a generalization of [1, Theorem 4].

Corollary 7 Let $R$ be a 2-torsion free prime ring with involution of the second kind and $(0) \neq I$ be $a *$-ideal of $R$. If $G$ is a generalized derivation of $R$ with associated derivation $\delta$ such that $x \circ x^{*} \pm \nabla\left[x, G\left(x^{*}\right)\right] \in Z(R)$ for all $x \in I$, then $R$ is commutative.

The following result is a generalization of [1, Theorem 5].

Corollary 8 Let $R$ be a 2-torsion free prime ring with involution of the second kind and $(0) \neq I$ be $a *$-ideal of $R$. If $F$ is a generalized derivation of $R$ with associated derivation $d$ such that $F(x) \circ x^{*}+\nabla\left[x, x^{*}\right] \in Z(R)$ for all $x \in I$, then $R$ is commutative.

\section{Acknowledgement}

We would like to thank the referee for his/her comments on the earlier draft of the manuscript. The research for the first author is supported by CSIRUGC (JRF, grant no. 402996).

\section{References}

[1] A. Abbasi, M.R. Mozumder and N.A. Dar, A note on skew Lie product of prime ring with involution, Miskolc Math. Notes, 21 (2020), no. 1, pp. 203-218. https://doi.org/10.18514/mmn.2020.2644

[2] A. Alahmadi, H. Alhazmi, S. Ali and A.N. Khan, Generalized derivations on prime rings with involution, Commun. Math. Appl., 8 (2017), no. 1, pp. 87-97.

[3] S. Ali, N.A. Dar and M. Asci, On derivations and commutativity of prime rings with involution, Georgian Math. J., 23 (2016), no. 1, pp. 9-14. https://doi.org/10.1515/gmj-2015-0016

[4] S. Ali, N.A. Dar and A.N. Khan, On strong commutativity preserving like maps in rings with involution, Miskolc Math. Notes, 16 (2015), no. 1, pp. 17-24. https://doi.org/10.18514/mmn.2015.1297 
[5] S. Ali, A.N. Khan and N.A. Dar, Herstein's theorem for generalized derivations in rings with involution, Hacettepe J. Math. Stat., 46 (2017), no. 6, pp. 1029-1034.

[6] H.E. Bell and M.N. Daif, On derivations and commutativity of prime rings, Acta Math. Hungar., 66 (1995), no 4, pp. 337-343. https://doi.org/10.1007/bf01876049

[7] H.E. Bell and N. Rehman, Generalized derivations with commutativity and anti-commutativity conditions, Math. J. Okayama Univ., 49 (2007), pp. 139-147.

[8] M. Brešar, On the distance of composition of two derivations to the generalized derivation, Glasgow Math. J., 33 (1991), pp. 89-93.

[9] M. Brešar, Centralizing mappings and derivations in prime rings, J. Algebra, 156 (1993), no. 2, pp. 385-394. https://doi.org/10.1006/jabr.1993.1080

[10] M.N. Daif, Commutativity results for semiprime rings with derivation, Internat. J. Math. Math. Sci., 21 (1998), no. 3, pp. 471-474.

[11] N.A. Dar and S. Ali, On *-commuting mappings and derivations in rings with involution, Turkish J. Math., 40 (2016), pp. 884-894. https://doi.org/10.3906/mat-1508-61

[12] N.A. Dar and A.N. Khan, Generalized derivations in rings with involution, Algebra Colloq., 24 (2017), no. 3, pp. 393-399. https://doi.org/10.1142/s1005386717000244

[13] I.N. Herstein, A note on derivations, Canad. Math. Bull., 21 (1978), pp. 369-370.

[14] M.A. Idrissi and L. Oukhtite, Some commutativity theorems for rings with involution involving generalized derivations, AsianEuropean J. Math., 12 (2019), no. 1, 1950001 (11 pages). https://doi.org/10.1142/s1793557119500013

[15] T.K. Lee, Generalized derivations of left faithful rings, Commun. Algebra, 27 (1999), no. 8, pp. 4057-4073. https://doi.org/10.1080/00927879908826682

[16] J. Ma, X.W. Xu and F.W. Niu, Strong commutativity-preserving generalized derivations on semiprime rings, Acta Math. Sinica, Eng. Ser., 24 (2008), no. 11, pp. 1835-1842. https://doi.org/10.1007/s10114-0087445-0 
[17] A. Mamouni, L. Oukhtite, B. Nejjar and J.J. Al Jaraden, Some commutativity criteria for prime rings with differential identities on Jordan ideals, Commun. Algebra, 47 (2019), no. 1, pp. 355-361. https://doi.org/10.1080/00927872.2018.1477945

[18] B. Nejjar, A. Kacha, A. Mamouni and L. Oukhtite, Commutativity theorems in rings with involution, Commun. Algebra, 45 (2017), no. 2, pp. 698-708. https://doi.org/10.1080/00927872.2016.1172629

[19] L. Oukhtite, Posner's second theorem for Jordan ideals in rings with involution, Expo. Math., 29 (2011), no. 4, pp. 415-419. https://doi.org/10.1016/j.exmath.2011.07.002

[20] G.S. Sandhu and D.K. Camci, Some results on prime rings with multiplicative derivations, Turk. J. Math., 44 (2020), no. 4, pp. 1401-1411. https://doi.org/10.3906/mat-2002-24

Bharat Bhushan

Department of mathematics,

Punjabi University,

Patiala 147002, Punjab, India.

bharat_rs18@pbi.ac.in

Gurninder Singh Sandhu

Department of mathematics,

Patel Memorial National College

Rajpura 140401, Punjab, India.

gurninder_rs@pbi.ac.in

Deepak Kumar

Department of mathematics,

Punjabi University,

Patiala 147002, Punjab, India.

deep_math1@yahoo.com

Please, cite to this paper as published in

Armen. J. Math., V. 13, N. 9(2021), pp. 11 18

https://doi.org/10.52737/18291163-2021.13.9-1-18 